ОСОБЕННОСТИ ПОРАЖЕНИЯ ГЛАЗ ПРИ ПЕРВИЧНОМ ГИПЕРПАРАТИРЕОЗЕ

( ) Н.Г. Мокрышева, Д.В. Липатов, Е.А. Добрева, Е.Е. Бибик*

ФГБУ «Национальный медицинский исследовательский центр эндокринологии» Минздрава России, Москва, Россия

Патология органа зрения встречается при различных эндокринных заболеваниях, однако сведения о состоянии зрительного аппарата при первичном гиперпаратиреозе (ПГПТ) до настоящего времени остаются ограниченными. Крупные систематизированные исследования по данной проблеме отсутствуют, в основном в литературе представлены описания отдельных клинических случаев, свидетельствующих о неспецифических изменениях со стороны глаз при развитии тяжелой гиперкальциемии. К наиболее частым проявлениям гиперпаратиреоидной офтальмопатии относят кальцификацию роговицы и конъюнктивы, нарушение функции глазодвигательных мышц. Принимая во внимание преобладание мягких форм ПГПТ, обусловленное ранней диагностикой и своевременным началом патогенетического лечения заболевания, в настоящее время данные нарушения встречаются редко. Тем не менее, специфическое поражение глаз у данной категории пациентов требует углубленного изучения и проведения дальнейших исследований.

КЛЮЧЕВЫЕ СЛОВА: первичный гиперпаратиреоз, гиперкальциемия, кальцинированная ленточная кератопатия, кальцификация.

\title{
FEATURES OF THE EYE PATHOLOGY IN PRIMARY HYPERPARATHYROIDISM
}

\author{
(c) Nataya G. Mokrysheva, Dmitry V. Lipatov, Ekaterina A. Dobreva, Ekaterina E. Bibik*
}

Endocrinology research centre, Moscow, Russia

The pathology of vision occurs in various endocrine diseases, but information about the state of the eyes in patients with primary hyperparathyroidism (PHPT) has been limited yet. Large systematic studies on this problem are absent, mainly the data of foreign literature are based on descriptions of several clinical cases that report about non-specific changes of eyes in people with significant hypercalcemia. The most common manifestations of hyperparathyroid ophthalmopathy are the cornea and conjunctiva calcification, disfunction of the eye muscles. Taking into account the prevalence of mild PHPT caused by early diagnosis and timely pathogenetic treatment of the disease, nowadays these ophthalmic disorders are rare. However, specific eye pathology in this category of patients requires detailed study and further research.

KEYWORDS: primary hyperparathyroidism, hypercalcemia, calcific band keratopathy, calcification.

\section{ВВЕДЕНИЕ}

Первичный гиперпаратиреоз (ПГПт) - заболевание, связанное с избыточной продукцией паратиреоидного гормона (ПТГ) околощитовидными железами и вовлекающее в патологический процесс различные органы и системы организма, что приводит к существенному снижению качества жизни, а также инвалидизации пациентов [1]. Данные о состоянии зрительного аппарата при ПГПТ до настоящего времени остаются ограниченными. K наиболее частым проявлениям гиперпаратиреоидной офтальмопатии относят кальцификацию роговицы с развитием кальцинированной ленточной кератопатии, кальцификацию конъюнктивы, нарушение функции глазодвигательных мышц [2-4]. Крупные систематизированные исследования по изучению данной проблемы отсутствуют, в основном в литературе представлены описания отдельных клинических случаев.

\section{КАЛЬЦИНИРОВАННАЯ ЛЕНТОЧНАЯ КЕРАТОПАТИЯ}

Кальцинированная ленточная кератопатия - изменение роговицы, развивающееся вследствие хронической гиперкальциемии различной этиологии. Она характеризуется отложениями кальция в боуменовой мембране, базальной мембране эпителия роговицы, наиболее по- верхностных слоях стромы, делая ее непрозрачной. Поражение поверхностных структур - основное ее отличие от петрификации роговицы, крайне редкой патологии, при которой, как правило, поражаются более глубокие слои передней части склеры. Помутнение белого или сероватого цвета развивается в межпальпебральной области, начинаясь от периферии в виде горизонтальных тяжей. Оно отделено от лимба тонкой прозрачной зоной и содержит неповрежденные участки роговицы в местах, где нервы пенетрируют боуменову мембрану, придавая вид «швейцарского сыра» $[5,6]$.

Точный механизм развития кальцинированной ленточной кератопатии до сих пор остается неизвестным. Отложение солей кальция (гидроксиапатита) в роговице связано с внешними факторами, так как дегенеративный процесс развивается в области, подверженной воздействию внешней среды. Это может быть результатом испарения слезной жидкости, изменения ее осмолярности, повышения рН в ходе метаболических процессов или локального увеличения концентрации ионов кальция и фосфата, превышающего порог растворимости элементов. В физиологических условиях значение $\mathrm{pH}$ межпальпебральной области выше, чем в остальной части роговицы, что связано с выделением углекислого газа с поверхности оболочки глаза. Суммарный эффект нескольких буферных систем крови препятствует коле- 
баниям кислотно-основного равновесия в местах с наиболее развитой васкуляризацией. Вероятно, данный механизм объясняет наличие четких прозрачных участков роговицы, имеющих развитое лимбальное кровообращение. Отсутствие поражения глубоких слоев стромы роговицы может быть обусловлено особенностями местного метаболизма - накоплением молочной кислоты в ходе анаэробного гликолиза и, как следствие, снижением рН среды. Кроме того, особое значение имеет анатомическое строение базального слоя эпителия и боуменовой мембраны. Отличием кератопатии при системной гиперкальциемии от дегенеративных и поствоспалительных изменений является первичное расположение кальциевых отложений интрацеллюлярно, а не вне эпителиальных клеток [5].

M. Petrochelos и соавт. представили случай тяжелого течения ПГПТ у 24-летней женщины. Заболевание дебютировало в послеродовом периоде с развитием выраженной анорексии, двусторонней глухоты и снижением зрения [7]. Выявленная ленточная кератопатия позволила заподозрить наличие гиперкальциемии, а гистологическое исследование тканей роговицы и конъюнктивы, полученных при биопсии из области лимба, подтвердило отложение кристаллов кальция. По данным лабораторного анализа отмечалось повышение уровня сывороточного кальция до 4,05 ммоль/л, и гиперкальциурия до 15,6-19,8 ммоль/сут. Была визуализирована аденома околощитовидной железы 1,5×1,0 cм, удаленная в ходе паратиреоидэктомии. При обследовании через месяц после операции наблюдалось восстановление остроты зрения и слуха, значимое улучшение общего самочувствия. Проявления ленточной кератопатии значимо регрессировали с восстановлением прозрачности роговицы обоих глаз в области зрачка и сохранением лишь ограниченных участков помутнения около 2 мм в области лимба.

Развитие ленточной кератопатии довольно редко приводит к нарушению зрения и требует лечения в отдельных случаях при появлении чувства дискомфорта, инородного тела в результате механического раздражения оболочки глаза избыточным отложением гидроксиапатита кальция, фотофобии или формировании язв роговицы, выраженном снижении остроты зрения. Рассматриваются различные подходы к лечению, включая хирургическое удаление, хелатирующую терапию, эксимерную лазерную фоторефракционную кератэктомию и как дополнительный метод для обеспечения скорейшего заживления и сохранения стабильности поверхности роговицы - пересадку амниотической мембраны [6].

\section{КАЛЬЦИФИКАЦИЯ ОБОЛОЧЕК ГЛАЗА}

Опубликовано несколько работ с описанием кальцификации слизистой оболочки глаза (конъюнктивы) при гиперпаратиреозе. D.K. Lee и A.K. Eiferman первыми представили клинический случай 34-летней женщины с длительным анамнезом ПГПТ в течение 10-15 лет и последующей паратиреоидэктомией [3]. В течение двух месяцев пациентка отмечала покраснение и чувство инородного тела в глазах. При сохранной остроте зрения были выявлены значительная инъекция конъюнктивы глазных яблок и кальцификация маргинального края века с двух сторон. Помимо этого, наблюдались ленточная кератопатия и отложение кальция в области лимба. С учетом выраженного чувства дискомфорта было произведено удаление кальцификатов роговицы и конъюнктивы, при гистологическом исследовании подтверждено наличие солей кальция в субэпителиальных слоях. Подобные поражения конъюнктивы глаза также отмечены при вторичном и третичном гиперпаратиреозе $[8,9]$.

Ph. Finny и соавт. описывают пациента 38 лет, первоначально обратившегося к офтальмологу с жалобами на покраснение глаз на фоне повышенной утомляемости и общей слабости [10]. При обследовании были выявлены билатеральный склерит и ретинальные геморрагии. Признаки инфекционного и воспалительного поражения глаз отсутствовали. При двукратном биохимическом исследовании крови зарегистрированы выраженная гиперкальциемия (3,22-3,77 ммоль/л) в сочетании с гипофосфатемией (0,67 ммоль/л), повышением активности щелочной фосфатазы до 932 Ед/л (референсные значения 40-120). Уровень сывороточного паратгормона составил 2770 нг/л (референсные значения 12-72). Таким образом, диагноз ПГПТ не вызывал сомнений. При дообследовании обнаружена аденома левой нижней околощитовидной железы по данным ультразвукового и радиологического исследований. После проведения паратиреоидэктомии синдром «красного глаза» регрессировал.

Отложение кристаллов кальция может происходить и в сосудистой оболочке глаза, классифицируемое как склеро-хориоидальная кальцификация (СХK). При этом образуются плоские или выпуклые бляшки, внешне сходные с такими образованиями, как хориоидальный невус, меланома, лимфома, хориоидальная остеома, метастазы. Обычно они имеют желтый или белый цвет и располагаются в верхне-височном квадранте или ближе к экватору. В области поражения отмечается атрофия хориоидеи и пигментного эпителия сетчатки, при этом не характерно наличие кровоизлияний, скоплений жидкости в субретинальных слоях, неоваскуляризации. От злокачественной патологии дегенеративный процесс отличают стабильный характер течения и минимальный риск потери зрения. Данные клинические признаки CXK описали C.L. Shields и соавт. у 118 пациентов (средний возраст 69 лет), наблюдавшихся у них в офтальмологическом госпитале, при обследовании 179 глаз выявив связь с системными нарушениями кальциевого обмена. Так, в 27\% случаев СХК диагностирована среди пациентов с ПГПТ [11].

\section{ПОРАЖЕНИЕ ГЛАЗОДВИГАТЕЛЬНЫХ МЫШЦ}

Мышечная слабость - один из наиболее частых симптомов гиперфункции околощитовидных желез. При ПГПТ может развиваться поражение различных мышц, в том числе глазного яблока, имеющих в составе волокна скелетной мускулатуры. В.H. Forman и соавт. опубликовали клинический случай пациента, страдающего косоглазием с развитием диплопии вследствие ПгПТ [12]. Патология головного мозга и орбит была исключена. Несмотря на гиперкальциемию 12,5 мг/дл (3,12 ммоль/л), ленточная кератопатия и слабость проксимальной мускулатуры отсутствовали. Установить 
связь между снижением тонуса экстраокулярных мышц и патологией околощитовидных желез исследователям удалось вследствие полного регресса глазной симптоматики на фоне достижения нормокальциемии после паратиреоидэктомии.

\section{ДРУГАЯ ПАТОЛОГИЯ ОРГАНА ЗРЕНИЯ}

В литературе описаны редкие случаи асимметричного экзофтальма, развившегося у пациентов с ПГПТ и наличием «бурых опухолей» костей черепа $[13,14]$. Натяжение или компрессия зрительного нерва при проптозе (экзофтальме) потенциально может приводить к нарушению фокусировки, цветового восприятия или к прогрессирующей потере зрения.

Ишемическая невропатия зрительного нерва с постепенным снижением остроты зрения (до счета пальцев у лица) как возможное осложнение гиперкальциемии и гиперпаратиреоза, была представлена D. Chappel и K. Farrington у пациентки 58 лет с повышением уровня сывороточного кальция до 3,66 ммоль/л и аденомой левой нижней околощитовидной железы (2,5×3,5×2,0 см) [15]. Ремиссия ПГПТ после хирургического лечения не привела к восстановлению зрительной функции, что вероятно связано с необратимой полной атрофией зрительного нерва. В качестве возможных механизмов ишемического повреждения нервных волокон рассматривается развитие микротромбозов, риск которых повышен при гиперкальциемии и сопутствующей дегидратации, кальцификации питающих сосудов, ар- териальной гипертензии и спазме церебральных артерий. Не исключается и прямое действие ПтГ на нервную ткань.

В 2016 г. Н. Baser и соавт. проведено исследование по изучению толщины роговицы (измеренной по центру), толщины сетчатки и внутриглазного давления у больных ПГПТ. Значимых отличий по сравнению со здоровым контролем выявлено не было. Статистически значимой ассоциации между уровнями ПТГ и кальция сыворотки крови с обозначенными параметрами также не установлено [16].

\section{ЗАКЛЮЧЕНИЕ}

При тяжелом течении ПгПт описаны случаи неспецифического поражения различных структур глаза, вероятнее всего, обусловленные развитием тяжелой гиперкальциемии. Однако, не исключается и прямое действие ПтГ на нервную и мышечную ткань. Принимая во внимание преобладание мягких форм ПГПТ, обусловленное ранней диагностикой и своевременным началом патогенетического лечения заболевания, в настоящее время данные нарушения встречаются редко. Тем не менее, требуется углубленное изучение патогенетических механизмов поражения глаз у пациентов с ПГПТ, а также проведение крупных систематизированных исследований по данной проблеме.

Конфликт интересов. Авторы декларируют отсутствие явных и потенциальных конфликтов интересов, связанных с публикацией настоящей статьи.

\section{СПИСОК ЛИТЕРАТУРЫ | REFERENCES}

1. Мокрышева Н.Г. Первичный гиперпаратиреоз. Эпидемиология клиника, современные принципы диагностики и лечения: Дис. . докт. мед. наук - Москва; 2011. [Mokrysheva NG. Pervichnyi giperparatireoz. Epidemiologiya, klinika, sovremennye printsipy diag nostiki i lecheniya. [dissertation] Moscow; 2011. (In Russ.)] Доступно по: http://www.dslib.net/luch-diagnostika/pervichnyj-giperparatireoz-jepidemiologija-klinika-sovremennye-principy-diagnostiki.html Ссылка активна на 09.04.2019.

2. Lee DB, Zawada ET, Kleeman CR. The pathophysiology and clinical aspects of hypercalcemic disorders. West J Med. 1978;129(4):278-320. PMID:362722

3. Lee DK. Ocular Calcifications in Primary Hyperparathyroidism. Arch Ophthalmol. 2006;124(1):136-137. doi: 10.1001/archopht.124.1.136

4. Jacob J, Chopra R, Chander A. The eye as a window to rare endocrine disorders. Indian J Endocrinol Metab. 2012;16(3):331-338. doi: 10.4103/2230-8210.95659

5. O'Connor GR. Calcific band keratopathy. Trans Am Ophthalmol Soc. 1972;70:58-81. PMID: 4677022.

6. Jhanji V, Rapuano CJ, Vajpayee RB. Corneal calcific band keratopathy. Curr Opin Ophthalmol. 2011;22(4):283-289. doi: 10.1097/ICU.0b013e3283477d36

7. Petrohelos M, Tricoulis D, Diamantacos P. Band keratopathy with bilateral deafness as a presenting sign of hyperparathyroidism. $\mathrm{Br} J$ Ophthalmol. 1977. doi: 10.1136/bjo.61.7.494

8. Berkow JW, Fine BS, Zimmerman LE. Unusual ocular calcification in hy perparathyroidism. Am J Ophthalmol. 1968;66(5):812-824. PMID: 5686911
9. Moutopoulos Canellos H, Cooper J, Paek A, Chien J. Multiple calcified deposits along the eyelid margins secondary to chronic renal failure and hyperparathyroidism. Optom - J Am Optom Assoc 2005;76(3):181-184. doi: 10.1016/S1529-1839(05)70283-9

10. Finny P, Jacob JJ, Thomas N. Primary hyperparathyroidism presenting as redness of eyes. Eur J Intern Med. 2007;18(8):608 doi: 10.1016/j.ejim.2007.03.005

11. Shields CL, Hasanreisoglu M, Saktanasate J, Shields PW, Seibel I, Shields JA. SCLEROCHOROIDAL CALCIFICATION. Retina. 2015;35(3):547-554. doi: 10.1097/IAE.0000000000000450

12. Forman BH, Ciardiello K, Landau SJ, Freedman JK. Diplopia associated with hyperparathyroidism: report of a case. Yale J Biol Med. 1995;68(5-6):215-217. PMID: 8903046

13. Block MB, Johnston $H$, Kartub MG. Unilateral exophthalmos: an unusual presentation of primary hyperparathyroidism. Ariz Med. 1978;35(12):785-786. PMID: 727953

14. Jahagirdar VR, Gittoes NJ. A rare presentation of primary hyperparathyroidism [abstract]. Endocr Abstr. 2015;38. P14 doi: 10.1530/endoabs.38.P14

15. Chappel D, Farrington K. Primary hyperparathyroidism presenting as unilateral visual loss. Postgrad Med J. 1991;67(787):469-470. doi: 10.1136/pgmj.67.787.469

16. Baser $\mathrm{H}$, Cuhaci $\mathrm{N}$, Topaloglu $\mathrm{O}$, et al. Is there any association between primary hyperparathyroidism and ocular changes, such as central corneal thickness, retinal thickness, and intraocular pressure? Endocrine. 2016;51(3):545-550. doi: 10.1007/s12020-015-0724-5

\section{ИНФОРМАЦИЯ ОБ АВТОРАХ [AUTHORS INFO]}

*Бибик Екатерина Евгеньевна [Ekaterina E. Bibik, MD, postgraduate student]; адрес: Россия, 117036, Москва, улица Дм. Ульянова, д.11 [address: 11 Dm. Ulyanova street, 117036 Moscow, Russia]; ORCID: https://orcid.org/0000-0001-5952-5846; eLibrary SPIN: 8522-9466; e-mail: bibikaterina@mail.ru 
Мокрышева Наталья Георгиевна, д.м.н., профессор РAH [Natalya G. Mokrysheva, MD, ScD, Professor of RAS];

ORCID: http://orcid.org/0000-0002-9717-9742; eLibrary SPIN: 5624-3875; e-mail: nm70@mail.ru

Липатов Дмитрий Валентинович, д.м.н. [Dmitry V. Lipatov, MD, ScD]; ORCID: https://orcid.org/0000-0002-2998-3392;

eLibrary SPIN: 9601-3993; e-mail: glas1966@rambler.ru

Добрева Екатерина Александровна, к.м.н. [Ekaterina A. Dobreva, MD, PhD];

ORCID: https://orcid.org/0000-0002-8916-7346; eLibrary SPIN: 3405-2467; e-mail: dobrevae@mail.ru

\section{ЦИТИРОВАТЬ:}

Мокрышева Н.Г., Бибик Е.Е., Липатов Д.В., Добрева Е.А. Особенности поражения глаз при первичном гиперпаратиреозе // Ожирение и метаболизм. - 2019. - Т.16. - №1. - C. 3-6. doi: 10.14341/omet9787

\section{TO CITE THIS ARTICLE:}

Mokrysheva NG, Lipatov DV, Dobreva EA, Bibik EE. Features of the eye pathology in primary hyperparathyroidism. Obesity and metabolism. 2019;16(1):3-6. doi: 10.14341/omet9787 